\title{
Aux origines du terminal
}

Les mutations techniques du transbordement des vracs dans les ports de commerce français au cours de l'entre-deux-guerres

The origins of the terminal. Technical changes in bulk transshipment in French commercial ports during the inter-war period

\section{Bruno Marnot}

\section{(2) OpenEdition}

\section{Journals}

Édition électronique

URL : https://journals.openedition.org/artefact/9924

DOI : 10.4000/artefact.9924

ISSN : 2606-9245

Éditeur :

Association Artefact. Techniques histoire et sciences humaines, Presses universitaires du Midi

Édition imprimée

Date de publication : 7 octobre 2021

Pagination : 149-176

ISBN : 978-2-8107-0755-3

ISSN : 2273-0753

\section{Référence électronique}

Bruno Marnot, «Aux origines du terminal », Artefact [En ligne], 14 | 2021, mis en ligne le 07 octobre 2021, consulté le 07 octobre 2021. URL : http://journals.openedition.org/artefact/9924 ; DOI : https:// doi.org/10.4000/artefact.9924

\section{(c) (†) $\odot$}

Artefact, Techniques, histoire et sciences humaines est mise à disposition selon les termes de la Licence Creative Commons Attribution - Pas d'Utilisation Commerciale - Pas de Modification 4.0 International. 


\title{
Aux origines du terminal
}

\author{
Les mutations techniques du transbordement \\ des vracs dans les ports de commerce français \\ au cours de l'entre-deux-guerres
}

\section{Bruno Marnot}

\section{Résumé}

Les années de l'entre-deux-guerres ont constitué une nouvelle étape dans la rationalisation du travail portuaire. Les nouveaux gains de productivité n'étaient plus recherchés, comme auparavant, dans la seule adaptation des grues aux navires, mais aussi désormais aux marchandises. Les diverses matières pondéreuses (charbon, céréales, pétrole, etc.) transportées en vrac furent le terrain d'expérimentation de nouveaux engins de transbordement et d'entrepôts spécialisés. Le terme de ce processus fut la spécialisation des espaces de travail qui allait donner naissance au principe du terminal. Les mutations techniques des grands ports de commerce français constituent une étude de cas exemplaire de cette évolution.

\section{Mots-clés}

entrepôts, grues, pipelines, ports, terminal portuaire, transbordement, transporteurs

99 Bruno Marnot, « Aux origines du terminal. Les mutations techniques du transbordement des vracs dans les ports de commerce français au cours de l'entre-deuxguerres », Artefact, 14, 2021, p. 149-176. 


\section{The origins of the terminal}

Technical changes in bulk transshipment in French commercial ports during the inter-war period

\section{Abstract}

The interwar period constituted a new stage in the rationalization of port work. New productivity gains were no longer sought, as before, in the mere adaptation of cranes to ships, but now also to goods. The various heavy goods (coal, wheat, oil, etc.) transported in bulk were the testing ground for new transshipment equipment and specialized warehouses. The end of the process was the specialization of the working spaces which gave birth to the terminal principle. The technical mutations of the major French commercial ports provide an exemplary case study of this evolution.

\section{Keywords}

conveyors, cranes, pipelines, ports, port terminal, transshipment, warehouses

\section{Introduction}

Largement entamée dans la deuxième moitié du XIX ${ }^{e}$ siècle, la mécanisation du transbordement franchit un nouveau saut technique à la suite de la Première Guerre mondiale. Le XIX ${ }^{\mathrm{e}}$ siècle a été celui de l'avènement des grues mues par les moteurs issus de la révolution industrielle : d'abord à vapeur, puis hydrauliques, enfin de plus en plus à l'électricité à partir des années 1880. Ce "mix énergétique » est présent dans les grands ports français avant 1914 et même après. Néanmoins, sur les quais comme dans l'activité manufacturière, l'avenir appartient à l'énergie électrique et à ses nombreux atouts : son infinie divisibilité, un meilleur rendement, une souplesse d'utilisation sans égale et un potentiel automobile qui permet le déplacement facile des grues le long des quais. C'est également l'électricité 
qui rend possible la mise au point de systèmes de transbordement spécialisés aux différents types de vrac, comme le montrent les premières applications qui ont lieu au moment où se déclenche la deuxième révolution industrielle ${ }^{1}$. Par conséquent, la mécanisation du transbordement connaît une double accélération au cours de l'entre-deux-guerres. D'une part, la force motrice se développe dans des proportions considérables, à la fois de manière intensive, par l'augmentation de la puissance installée par unité de traitement, et de manière extensive par la généralisation des engins. Traduite en termes économiques, cette tendance souligne l'intensification du facteur capital dans le cadre du travail portuaire. D'autre part, si les grues connaissent de nouvelles améliorations, c'est surtout du côté de l'outillage spécialisé que se produisent les innovations majeures. Cette analyse sera menée à partir de l'étude des ports français dont l'évolution technique reflète celle des autres grands organismes mondiaux, en particulier européens et nord-américains, qui s'est produite au même moment. Toutefois, le cas français présente un intérêt particulier en raison du rôle d'accélérateur joué par la Première Guerre mondiale, comme nous le verrons dans un premier temps. Le deuxième temps s'appesantira sur les caractéristiques de l'automatisation croissante des opérations de manutention des marchandises pondéreuses. Précisons enfin que notre analyse privilégiera la séquence de transbordement mer-terre, où les innovations sont plus décisives que celles qui ont lieu entre le transport maritime et le transport fluvial.

\section{Le rôle accélérateur de la guerre}

\section{Mécanisation à outrance et hausse des rendements}

À maints égards, la Première Guerre mondiale a représenté un tournant majeur dans l'histoire contemporaine des ports de commerce français. Elle fut un moment de vérité et un événement fondateur. Moment de vérité car les ports français ont dû alimenter à la fois l'effort de guerre national, soutenir celui de ses alliés britanniques engagés sur le sol français et assurer le ravitaillement de la population. Moment fondateur car les pouvoirs 
publics ont pris conscience du rôle stratégique des ports. Alors que leurs trafics sont devenus essentiellement à sens unique, leurs horizons maritimes respectifs se sont restreints aux relations avec le Royaume-Uni et les ÉtatsUnis. De fait, les ports de l'Atlantique, depuis la Normandie jusqu'au SudOuest, ont été les plus sollicités. Au moment des premiers engagements militaires, à l'été 1914, les ports n'étaient pas plus que les autres secteurs de l'économie préparés à une guerre longue. L'entrée dans un conflit plus long que prévu à partir de 1915 a rendu leur situation critique, de façon aussi subite que rapide. Sous la pression de l'urgence, les lourds et longs travaux d'infrastructure, exigeants en moyens humains et en ressources financières, furent sacrifiés dans la plupart des cas. Pour réduire autant que possible les goulets d'étranglement, les efforts furent menés de façon simultanée dans trois directions : créer des appontements pour multiplier les postes d'accostage ; augmenter le nombre d'engins de levage et des lieux de stockage ; améliorer enfin les capacités d'évacuation des marchandises.

L'un des aspects spectaculaires de l'équipement des ports pendant la guerre résida dans la démultiplication des kilomètres de rails - 350 kilomètres supplémentaires entre 1914 et $1917^{2}$ - et des appareils de levage. "Les pouvoirs publics durent donc pourvoir à la main-d'œuvre, compléter un outillage trop souvent archaïque, et presque toujours incomplet, développer les postes de déchargement, enfin créer les moyens d'évacuation qui manquaient ", résuma Auguste Pawlowski ${ }^{3}$. On doit à ce chartiste et journaliste passionné par la chose maritime d'avoir dressé la chronique des ports français en guerre dans une série d'articles bien documentés qui furent publiés par la revue du Génie civil. Selon lui, « les appareils de déchargement constituaient incontestablement le point faible de nos ports dans la majorité des $\operatorname{cas}^{4}{ }$. Comptant un parc de 1240 engins de levage en août 1914, les ports en exploitaient 300 de plus trois ans plus tard, tandis que 100 autres étaient en cours de construction ou de montage. L'auteur notait en outre que " les appareils nouveaux [étaient] d'un rendement très supérieur aux anciens et que la plupart [étaient] à bennes automatiques. En outre, beaucoup d'élévateurs à grains ont été installés, dont le débit est

2. Claveille, 1919. Je remercie Damien Mouries pour la communication de ce document.

3. Pawlowski, 1917, p. 343.

4. Pawlowski, 1917, p. 344. 
considérable ${ }^{5}$ ". Les nouveaux engins de levage acquis par les ports étaient de construction britannique ou américaine.

Les efforts d'équipements furent concentrés sur les organismes considérés d'un intérêt logistique stratégique pour le ravitaillement de la France en guerre. Pas moins de 165 appareils de levage furent établis à Saint-Nazaire, en particulier pour accélérer le transbordement du charbon qui représentait environ la moitié du tonnage de son trafic total ${ }^{6}$. Rouen disposait en 1914 de 87 grues de quai et de 80 pontons-grues représentant une puissance totale de levage de 409 tonnes. Au moment de l'armistice, leurs nombres respectifs était de 117 et 109, pour une force de 619 tonnes. En outre, le réseau des Chemins de fer de l'État mit en service à partir de 1917 deux grands transbordeurs électriques pour la manutention rapide des charbons. Ces appareils pouvaient prendre d'un coup 5 tonnes de charbon dans les cales des navires pour les transférer dans les chalands et les wagons ou bien les déposer dans les silos de stockage. Leur rendement pouvait varier de 85 à 200 tonnes par heure selon la nature des charbons manutentionnés ${ }^{7}$.

Un matériel plus nombreux et puissant se traduisit par des rendements croissants dans la manutention des marchandises, surtout des matières pondéreuses. Les relevés quotidiens du Ministère des Travaux publics établissaient que les vitesses moyennes de débarquement du charbon dépassaient 800 tonnes par jour à Rouen, Saint-Malo, Rochefort, Bayonne, et 700 tonnes à Brest, Saint-Nazaire, Nantes, La Pallice, Bordeaux et Marseille. À Rouen, qui était le principal nœud portuaire logistique de la France en guerre, 682000 tonnes de houille purent être déchargées au cours du mois de mai 1917, alors qu'au meilleur de l'année 1913 on n'avait jamais dépassé 270000 tonnes. Cela équivalait à une hausse du rendement de l'ordre de $150 \%{ }^{8}$. Dans un rapport de 1919 , le ministre des Travaux Publics Albert Claveille, qui a joué un rôle déterminant dans l'organisation logistique de l'effort de guerre, dressa le bilan des améliorations apportées dans le secteur portuaire. Entre 1914 et 1917, la puissance instantanée de déchargement fut accrue de $85 \%$ et 153 nouveaux postes à quai furent mis en service dont 120 pour les seuls navires de mer $^{9}$. Malgré

5. Pawlowski, 1917, p. 344.

6. Barbance, 1949, p. 149.

7. Croguennec, 1999, p. 244-251.

8. Pawlowski, 1917, p. 144.

9. Claveille, 1919. 
les difficultés de toutes sortes, le défi du rendement fut relevé et atteignit dans les ports français des niveaux bien supérieurs à ceux de l'avant-guerre.

\section{Le temps des ports « usines"}

Longtemps ignorée par les ingénieurs des Ponts et Chaussées ${ }^{10}$, la notion de rendement du travail portuaire gagna progressivement quelques esprits lucides dans les dernières années du XIX ${ }^{\mathrm{e}}$ siècle lorsque les milieux d'affaires maritimes, certains dirigeants politiques et la technocratie des Travaux Publics prirent conscience du décrochage des principaux établissements français par rapport à leurs voisins européens. Les ports de la Manche notamment, tels que Le Havre et Dunkerque, souffraient ouvertement de la concurrence d'Anvers et de Hambourg. En 1912, Les ingénieurs Laroche et Pobeguin présentèrent au quatrième congrès national des travaux publics français une «étude comparée de l'outillage des ports français et étrangers " qui, à l'aide de nombreux indicateurs, démontraient l'infériorité des principaux établissements français ${ }^{11}$. Leur moindre productivité était en partie cause de leur relative cherté.

C'est au cours du même congrès que l'ingénieur Charles Viguerie compara les grands ports à des " organismes industriels régis par les mêmes lois que l'industrie elle-même ${ }^{12}$. Ces lois, indispensables pour rendre les ports compétitifs, reposaient, d'une part, sur " l'aménagement général du port ", c'est-à-dire son organisation rationnelle et, d'autre part, sur « son outillage de manutention et de déchargement ». L'auteur abondait dans le sens de ses deux collègues ingénieurs quant à l'insuffisance de l'outillage, tant en nombre d'unités de transbordement qu'en puissance de levage. Quatre préconisations ressortaient de sa réflexion : l'outillage comme « indispensable trait d'union » entre les navires et les magasins ; l'augmentation de la portée des grues ; l'emploi des " transporteurs" pour les marchandises en vrac ; la généralisation de l'électricité comme force motrice ${ }^{13}$. Au moment de la deuxième révolution industrielle, l'analogie entre le port et l'usine prenait du sens. Lewis Mumford et David Landes ont énoncé les principales caractéristiques de l'usine : une unité de production qui utilisait une source

10. Voir sur ce sujet Marnot, 2011, chap. 4 "Ambitions et limites de l'expertise technique ».

11. Laroche et Pobeguin, 1912.

12. Viguerie, 1912.

13. Viguerie, 1912. 
d'énergie inanimée, rassemblant une communauté d'ouvriers travaillant au rythme des machines ${ }^{14}$. Car l'usine, à la différence de la manufacture pré-industrielle, était l'univers de la machine reine et de la productivité qui fixaient l'organisation et les conditions du travail ${ }^{15}$. La seule différence avec l'usine était que le port était appelé à devenir un immense organisme mécanique voué non pas à la production de biens mais à leur transbordement et leur conditionnement.

Cette vision du port-usine était centrale, par exemple, chez Georges Hersent, l'un des plus grands entrepreneurs de travaux portuaires de son temps et qui a produit plusieurs ouvrages et articles sur le sujet. Ainsi écrivait-il quelques années avant la Grande Guerre qu' " au point de vue industriel et économique, un port moderne n'est en réalité qu'une véritable usine de manutention, un organisme de suture entre les voies ferrées et les voies de navigation intérieure d'une part, et les grandes routes maritimes de l'autre ». Aussi appelait-il de ses vœux " une organisation industrielle bien appropriée " comme moyen d'abaisser les tarifs du port et d'attirer, par conséquent, un plus grand courant de marchandises ${ }^{16}$. En évoquant l'" usine de manutention " et "l'organisme de suture », Hersent avait en tête la chaîne de production en continu et in fine la fluidité du process. Et l'" organisation industrielle bien appropriée " était un appel à la rationalisation de l'espace et du travail portuaires. Ce point, l'entrepreneur le précisa dans un article postérieur à la guerre :

Dans nos sept grands établissements maritimes, qui pratiquent un trafic général et mélangé, la spécialisation devra se faire par quartiers ou secteurs du port. C'est un véritable scandale que les marchandises les plus diverses soient débarquées pêle-mêle sur les mêmes quais, avec des procédés identiques et un outillage insuffisant et uniforme. Un grand port, ce n'est pas une série ininterrompue de quais tous pareils, parsemés de grues plus ou moins nombreuses et toutes semblables. C'est pour ainsi dire la réunion de plusieurs ports spéciaux, à l'architecture et à l'outillage appropriés. Ce n'est qu'à cette condition que l'on réalisera la vitesse et le bon marché des opérations. Le caractère dominant

14. Mumford, 1934 ; Landes, 1975.

15. Voir sur ce sujet Musso, 2017, p. 567-569.

16. Hersent, 1908, p. 24. 
de ces installations spécialisées, ce sera leur industrialisation : et par industrialisation j'entends le développement à l'extrême de l'outillage et du machinisme ${ }^{17}$.

Cette longue citation est riche d'enseignements. Hersent soulignait, en premier lieu, que les principes de rationalisation dérivés de l'espace usinier devaient être portés en priorité sur les sept principaux ports français (Marseille, Le Havre, Rouen, Dunkerque, Nantes, Saint-Nazaire, Bordeaux), c'est-à-dire ceux qui moissonnaient plus de $80 \%$ du commerce extérieur de l'ensemble des établissements nationaux. Le deuxième enseignement réside dans la spécialisation spatiale des manutentions par catégories de marchandises. Autrement dit, Hersent définit, avant la lettre, le principe du terminal, qui est un port dans le port, disposant de sa propre autonomie fonctionnelle. Or, pour être pleinement efficace, le terminal doit adapter son outillage de transbordement à la marchandise. En dernier lieu, cet outillage spécifique doit se substituer le plus possible à la main-d'œuvre, afin d'augmenter la productivité du passage portuaire (transbordement-entreposage-évacuation).

En 1919, l'ingénieur en chef des Ponts et Chaussées, Victor Sabouret, qui fit l'essentiel de sa carrière à la Compagnie du chemin de fer ParisOrléans, donna une conférence retentissante devant les étudiants de son ancienne école ${ }^{18}$. Elle fit l'objet, quelques semaines plus tard, d'un copieux compte rendu dans la revue du Génie civil. Sabouret relatait son expérience des visites qu'il avait effectuées dans plusieurs ports américains, un peu à l'image des voyages d'étude qui avaient conduit, un siècle plus tôt, l'ingénieur Charles Dupin en Angleterre. Le parallélisme entre ces deux missions est intéressant à plus d'un titre. Dans chaque cas, les ingénieurs visitèrent des pays qui incarnaient les modèles techniques portuaires de leurs temps. Si Dupin avait vanté le principe révolutionnaire des docks-entrepôts édifiés à Londres et Liverpool, Sabouret rapporta ce qu'on pourrait qualifier de variante taylorienne des ports avec les "postes maritimes intensifs ».

Le modèle abouti, en l'occurrence, était celui des postes de transbordement du minerai de fer et du charbon. Sabouret notait d'abord que «l'organisation des ports américains [était] très différente de celle des établissements européens et surtout français " car ils se composaient, selon l'intéressant

17. Hersent, 1921, p. 42-43. Les termes en italiques sont de l'auteur.

18. Cette conférence est publiée dans Sabouret, 1919, p. 125. 
mot de l'auteur, d'une série de "terminus " appartenant à des sociétés privées, en particulier des compagnies ferroviaires qui étaient en concurrence les unes avec les autres. Chacun de ces " terminus " constituait donc une sorte de port dans le port. En outre, ces établissements particuliers étaient tous établis selon le même schéma organisationnel : un unique poste de déchargement, constitué d'une jetée perpendiculaire à la rive, muni de l'outillage le plus perfectionné et comportant, dans certains cas, un énorme réseau de voies ferrées ${ }^{19}$. La séquence de transfert du charbon entre terre et mer était entièrement automatisée grâce à la mise au point d'installations telles que les bennes culbuteuses (dumpers) et des wagons spéciaux de transbordement (transfer-car), comme l'ingénieur en vit dans le port de Norfolk (Virginie). À Baltimore, le transport du charbon entre le dumper et le navire était assuré par des courroies transporteuses qui aboutissaient à un grand portique d'embarquement mobile qui vidait le charbon dans une écoutille du navire ${ }^{20}$. C'est néanmoins dans les villes portuaires autour des Grands Lacs que se trouvaient les équipements les plus remarquables. Ainsi, dans le port de Cleveland, le transbordement du minerai était effectué au moyen de déchargeurs Hulett, qui étaient d'énormes grues à bras articulé, roulant sur des portiques. L'ensemble de l'installation nécessitait une considérable emprise au sol. Il existait donc bien un modèle américain du transbordement des pondéreux solides avec lequel les établissements français ne pouvaient rivaliser à la sortie de la Grande Guerre. L'enjeu consistait à « établir un nombre relativement restreint de postes fortement outillés, plutôt que des postes nombreux à rendement moyen ", bref il s'agissait, comme le résumait Sabouret, de remplacer « la méthode extensive par la méthode intensive ${ }^{21}$ ". L'organisation de " postes spécialisés » et tendant à l'automatisation intégrale des grands ports français répondait à une sorte d'" ardente nécessité ».

19. «L'outillage mécanique des ports... », 1920, p. 170-171.

20. "L'outillage mécanique des ports...", 1920, p. 172.

21. «L'outillage mécanique des ports...", 1920, p. 169. 


\section{Engins de levage : de la grue au portique}

\section{Les perfectionnements de la grue}

Les grues demeurèrent au cœur du dispositif de déchargement dans les ports de l'entre-deux-guerres. Comme on l'a vu, leur nombre s'était considérablement accru au cours du premier conflit mondial et continua d'augmenter par la suite. Dans le sillage des années d'avant-guerre, la disparité des sources d'énergie (vapeur, hydraulique, électricité) resta la règle. De même, prévalait la diversité des puissances de levage en fonction des besoins et la multiplicité des modèles (fixes, mobiles, flottantes, sur béquille, etc.), dans la mesure où les grues étaient aussi bien destinées au transbordement des matières pondéreuses qu'à celui des biens manufacturés. Ainsi, à Rouen, l'outillage public concédé à la chambre de commerce était-il disparate, mais les grues hydrauliques, trop petites et trop faibles, furent progressivement remplacées par des grues électriques au cours des années 1930. Les moteurs électriques concentraient tous les avantages par rapport aux autres formes d'énergie : les machines étaient plus simples d'emploi, plus fiables et donc d'un entretien moins onéreux. À la différence des grues hydrauliques, les grues électriques disposaient de leur propre moteur, ce qui entraînait une plus grande souplesse d'utilisation et rendait surtout possible leur déplacement automobile, sans recours au cabestan ${ }^{22}$.

La puissante dynamique d'équipement héritée du conflit se poursuivit au cours des deux décennies suivantes. À Rouen, toujours, la puissance cumulée de levage de l'ensemble du port, qui avoisinait, comme on l'a vu, 620 tonnes en 1918, avait doublé vingt ans plus tard (1 184 tonnes), pour un total de 293 engins (grues de quai, grues flottantes, portiques) ${ }^{23}$. Toutefois, l'augmentation de la charge de levage était inégale selon les catégories de marchandises manutentionnées. Elle était marginale dans le cas des marchandises diverses, en raison de la diversité des contenants (caisses, ballots, balles, etc.) qui exigeaient une intervention humaine de tout instant pour treuiller, charger ou décharger, puis acheminer les palanquées. La productivité de la manutention était, par conséquent, limitée à la force et à l'organisation des dockers. À la fin des années 1920, l'ingénieur en chef des Ponts et Chaussées Charles Laroche indiquait que le rendement

22. Borruey, 1994.

23. Croguennec, 1999. 
des appareils dédiés à la manutention des divers était en moyenne de 12,5 tonnes par heure contre 75 tonnes par heure pour des grues de 5 tonnes destinées au transfert des pondéreux en $\operatorname{vrac}^{24}$.

Ce fut davantage dans cette catégorie de fret que les progrès furent les plus manifestes, là où les bennes automatiques pouvaient remplacer la force de travail humain. Les bennes chargées à la main, à la fin du XIX siècle, disposaient d'une charge utile de 3 tonnes, tandis que celles qui étaient automatisées pendant l'entre-deux-guerres avaient non seulement doublé leur capacité de charge mais suivaient la loi des rendements croissants. C'était, par exemple, le cas de la grue cédée par la société M.A.N. que le port de Nantes reçut au titre des prestations en nature dues par l'Allemagne. Cet engin, d'une charge utile de 6 tonnes, prenait les minerais au moyen d'une benne à quatre câbles d'une capacité de $3 \mathrm{~m}^{3}$. Elle pouvait effectuer un double mouvement de rotation à $360^{\circ}$ et de translation le long d'un portique enjambant trois voies ferrées. Grâce à la puissance des moteurs électriques, la rapidité des mouvements permettait un rendement exceptionnel de 250 tonnes par heure, soit trois fois plus que le rendement normal des engins de 5 tonnes. Bien qu'utilisée en priorité pour les minerais, cette grue était polyvalente, puisque la benne pouvait être remplacée par une suspension à crochet pouvant supporter des charges unitaires de 12 tonnes ${ }^{25}$.

La hausse de la productivité du travail des grues ne dépendait pas seulement de l'augmentation de leur puissance de charge. La vitesse de travail, comme on l'a vu, joua un rôle déterminant, tout comme la hauteur et la portée maximales de leur flèche. L'augmentation de ces paramètres était conditionnée par la taille croissante des navires. L'une des grandes innovations fut la grue à flèche relevable - ou à portée variable - dont l'usage se généralisa au cours de l'entre-deux-guerres. La flexibilité de son envergure lui permettait de s'adapter à différents formats de navires. À Rouen, ces engins pouvaient, sans bouger, couvrir une surface de 27,60 mètres. À Marseille, cet outillage, construit en 1929 par les maisons associées EillersJeumont, fut installé sur le bassin du Président-Wilson. Lorsque les bras de ces grues étaient tendus à leur portée maximale, la poulie se trouvait suspendue à 14 mètres du quai et à une hauteur record de 26 mètres.

24. Laroche, 1931, p. 216.

25. "Grues sur portique pour le chargement des minerais... ", 1932, p. 463. 
Relevée au maximum, leur flèche atteignait une hauteur de 34 mètres, dépassant nettement les hangars en béton armé. La chambre de commerce de Marseille commanda une cinquantaine d'exemplaires de grues de ce type, de marque Applevage, pour équiper les quais de la gare maritime en $1936^{26}$. Installées majoritairement sur les quais, les grues à flèche relevable trouvèrent aussi leur place sur les navires. En 1931, la Société Demag en équipa les paquebots Caribia et Cordillera qui effectuaient des liaisons entre le port de Hambourg et le continent américain. Les organes mécaniques étaient enfermés dans des carters parfaitement étanches à l'eau de mer, tandis que les moteurs électriques et les freins électromagnétiques étaient cuirassés ${ }^{27}$.

\section{De la grue sur portique au portique à chariot}

A mesure que les grues gagnaient en mobilité et en dimension - et donc en poids -, les ingénieurs et constructeurs durent imaginer des supports à l'avenant. C'est ainsi que les grues furent disposées sur des demi-portiques, en appui à la fois sur l'arête du quai et sur le toit-terrasse du hangar. Elles firent, par exemple, leur apparition sur les quais de Marseille en 1904. Leur grand avantage par rapport aux grues traditionnelles sur pylônes était de désencombrer les quais. De plus, comme le précise René Borruey, avec les grues montées sur pylônes les deux éléments du dispositif de transbordement-entreposage étaient dissociés, alors que le demi-portique les intégrait et les rendait solidaires. L'équipement du bassin du Président-Wilson et la rénovation du bassin de la Joliette dans l'entre-deux-guerres obéirent à cette forme de modernisation des quais ${ }^{28}$. Les grues sur portique complet tendirent néanmoins à s'imposer dans l'entre-deux-guerres car le portique représentait un support plus stable pour des engins de grande envergure. De plus, le portique, à la différence du demi-portique, avait l'avantage d'être facilement déplacé d'un quai à l'autre. La gestion de ce type d'engin de levage était donc plus souple. Les grues sur portique étaient elles-mêmes diversifiées. À l'image de la grue M.A.N. évoquée plus haut, la grue pouvait rouler sur un pont métallique enjambant des voies de quai.

26. Borruey, 1994.

27. "Grues à volée variable à bord des navires ", 1938, p. 109.

28. Borruey, 1994. 
Un autre type apparaît plus décisif pour l'histoire des techniques de transbordement portuaire. Il s'agit des portiques à chariot apparus avant la Première Guerre mondiale pour le déchargement du charbon et qui se multiplièrent au cours des années 1920 et 1930. Ce fut le cas à Bordeaux, où la chambre de commerce en déploya sept juste après la guerre, sur la rive droite où se trouvait le nouveau port de la ville ${ }^{29}$. Toutefois, avant même le déclenchement du conflit, le transfert du charbon souffrait de l'insuffisance des capacités des déchargement, ce qui entraînait des retards et des surestaries ${ }^{30}$ à la charge des sociétés de manutention, ainsi que de l'encombrement des quais par la présence de nombreux faisceaux de voies en éventail. Il était donc impossible d'installer des transporteurs horizontaux à hauteur du sol. La voie aérienne restait la seule solution. Parmi les diverses propositions mises au concours, la chambre de commerce de Bordeaux opta pour le système proposé par la société «La Manutention Mécanique Industrielle ». Son projet s'inspirait des méthodes employées aux États-Unis dans les importantes installations de déchargement de minerai de la région des Grands Lacs. On peut aussi émettre l'hypothèse que le pont transbordeur d'Auguste Arnodin, qui avait connu son heure de gloire avant 1914, constituait une autre source d'inspiration plus ou moins consciente $^{31}$.

Les portiques implantés à Bordeaux étaient une intéressante combinaison de plusieurs techniques. Ils avaient l'allure de ponts transbordeurs mais aériens, puisque les rails de roulement se trouvaient à 20 mètres au-dessus du quai. Ils empruntaient évidemment au portique puisque leurs ponts roulants reposaient sur des jambages mobiles sur deux files de rails, l'une située sur le bord du quai et l'autre sur les silos. Sur les ponts roulants se déplaçaient des chariots-treuils qui supportaient des bennes preneuses de dimensions variables, dont les plus importantes de l'époque, qui avaient une charge utile de 6 tonnes. La longueur maximale de déplacement du chariot entre le navire et le silo était de 36 mètres. Le pont roulant au-dessus du quai était prolongé par une partie en porte-à-faux de 10 mètres au-dessus des navires. Cette partie pouvait d'ailleurs se relever, selon le principe de la flèche relevable, pour dégager, au besoin, les mâts des navires. Chaque

29. Marnot, 2012, p. 264-265.

30. Somme payée à l'armateur en dédommagement du dépassement de temps convenu par contrat pour le chargement ou le déchargement d'un navire.

31. Sur les ponts transbordeurs voir Marnot, 2015, p. 135-136. 
appareil était capable de décharger de 75 à 100 tonnes de matière pondéreuse à l'heure. À raison de trois appareils par navire, on pouvait compter sur une vitesse de déchargement de 225 tonnes à l'heure. L'ensemble des manœuvres - la descente à vide de la benne, son chargement automatique dans la cale du navire, le levage de la benne et le déplacement horizontal du chariot, la descente de la benne et son ouverture au-dessus de la trémie, et enfin son retour à vide au-dessus de la cale du navire - était réalisée par un machiniste qui pouvait, avec l'expérience, en produire en moyenne 60 par heure, soit une par minute.

Les bennes déversaient le charbon dans des trémies en forme de pyramide renversée. Chacune d'entre elles pouvait emmagasiner environ 90 tonnes de charbon, ce qui représentait un total de de 4400 tonnes de charbon pour les 48 trémies installées. Celles-ci étaient équipées de portes supérieures et inférieures qui mettaient leur contenu à l'abri des intempéries, en attendant leur déchargement dans les wagons par leur partie inférieure. Cette installation permettait aussi de desservir directement le parc à charbons de la Compagnie d'Orléans par un transporteur aérien, comme on le verra plus $\operatorname{loin}^{32}$.

Rouen, qui était le premier port charbonnier de France, fut également pionnier dans ce domaine. Les énormes quantités de houille traitées pendant le conflit décidèrent les autorités locales d'en équiper massivement la rive gauche, traditionnellement dédiée aux manutentions de pondéreux. En 1940, il possédait 21 portiques de 30 à 150 mètres de long et d'une force de levage totale de 173 tonnes. Les plus grands pouvaient opérer jusqu’à 34 mètres depuis l'arête du quai au-dessus du fleuve grâce à leur avant-bec relevable. Lorsque les cales des navires étaient parfaitement disposées et qu'il était donc possible d'affecter plus de deux portiques pour un seul navire, la vitesse de déchargement par poste pouvait atteindre 450, voire 600 tonnes par heure. Ces engins, particulièrement bien adaptés aux minerais en vrac, assuraient aussi le transbordement des bois ${ }^{33}$. Les portiques à chariot, qui combinaient mouvement vertical et horizontal, constituaient des engins hybrides entre les grues et les transporteurs. Ils étaient aussi, bien entendu, la préfiguration des futurs portiques à conteneurs. 


\section{Un impératif grandissant : s'adapter à la marchandise}

\section{La diversification des engins transporteurs}

L'effort de mécanisation des transbordements s'est porté en priorité sur les pondéreux pour deux raisons : d'une part, parce qu'ils représentaient l'essentiel des trafics portuaires ; d'autre part, parce que les transports maritimes et terrestres ont rendu possible leur transport en vrac, ce qui permettait d'imaginer plus facilement des dispositifs de transvasement que pour les biens manufacturés (les divers) aux conditionnements très disparates. La logique globale d'équipement des ports de commerce avant-guerre avait été commandée par l'augmentation de la taille des navires et la nécessité de réduire leur durée de stationnement à quai. Ce furent les paramètres essentiels de la "logique d'adaptation permanente " des ports ${ }^{34}$. Or les ports de l'entre-deux-guerres sont allés plus loin dans la rationalisation du travail en s'adaptant, non plus seulement au navire, mais à la marchandise. En matière d'outillage, mêmes les grues les plus perfectionnées montraient leurs limites opérationnelles. Cela signifiait donc que, comme l'avait montré le conflit, les organismes devaient intensifier l'usage des outillages de transbordement spécialisés, mais aussi des espaces de stockages spécialisés. In fine les infrastructures devaient à leur tour s'adapter. Au bout de cette logique pointait le principe du terminal avec ses postes de chargement / déchargement et sa chaîne de transmission des marchandises spécifiques, immédiatement identifiable dans l'espace portuaire.

À la différence des grues, qui étaient des engins de levage, caractérisés par un mouvement vertical et rotatif, les transporteurs obéissaient à un mouvement horizontal ou incliné, suivant une pente plus ou moins forte. La diversité de ces engins n'était que le reflet de celles des marchandises en vrac à transférer en reprise de charge directe (navire-transport terrestre) ou différée (navire-hangar-transport terrestre). Cette diversité était patente dans le domaine majeur du transbordement de minerais. C'était tout particulièrement vrai des transbordeurs de charbon par courroies, comme ceux qui furent mis en service à Dunkerque en $1917^{35}$. Des élévateurs à godet furent

34. Sur ce point voir Marnot, 2011, p. 63-99.

35. Pawlowski, 1919, p. 435-436. 
également installés, comme à Nantes, qui en installa huit pour le transfert des céréales; l'ensemble pouvait en transborder 1600 tonnes par jour ${ }^{36}$.

L’emploi des ponts, transbordeurs à bande et autres élévateurs ne se limita pas aux quais. Il gagna rapidement la profondeur des terre-pleins. Ainsi, à Bordeaux, des transporteurs aériens à câbles sillonnaient la plaine des Queyries pour desservir le parc à charbons de la Compagnie des chemins de fer d'Orléans qui était situé à 1500 mètres environ des appareils de déchargement. Ce parc était lui-même extrêmement vaste, puisqu'il s'étendait sur une longueur de 440 mètres et une largeur de 50 mètres. Utilisable sur une hauteur de 6 mètres, il pouvait recevoir jusqu'à 132000 mètres cubes de houille, soit environ 110000 tonnes. Les bennes suspendues aux câbles effectuaient la totalité du circuit de 4 kilomètres en un peu moins d'une heure. Lorsqu'elles arrivaient au-dessus du parc, les bennes déchargeaient leur cargaison, soit dans des trémies mobiles qui permettaient de réaliser le chargement direct des wagons, soit dans la trémie d'un pont transbordeur à courroie qui déversait à son tour le charbon en un point quelconque du parc. Dans ce cas, la reprise du charbon après stockage était effectuée au moyen d'un transporteur à bennes dont la voie de roulement était constituée non plus d'un câble mais d'un rail rigide. Le chariot portebenne se déplaçait le long de la poutre jusqu'au moment où il surplombait une trémie permettant le chargement des wagons. L'avantage de ce type de transporteur sur les engins à câbles résidait dans la simplification des opérations de maintenance que nécessitait l'entretien des filins d'acier. Il permettait, par conséquent, de substantielles économies d'exploitation ${ }^{37}$.

Le transport automatisé était dans certains cas la réponse à des contraintes techniques liées au produit. La Compagnie bordelaise des produits chimiques (CBPC) dota Nantes d'un complexe de production de superphosphates unique en Europe au milieu des années 1920. L'usine pouvait non seulement produire 120000 tonnes de superphosphates par an, mais aussi 10000 tonnes d'acide sulfurique nécessaires à leur fabrication et 10000 tonnes d'engrais composés. Ses expéditions quotidiennes pouvaient atteindre 2000 tonnes, soit 20000 sacs, pendant la campagne. Une fois défourné, le superphosphate était transporté dans les magasins de stockage, où il était mis en sac pour l'expédition. Or l'opération du

36. Kauffman, 1920, p. 91.

37. Martin, 3 juillet 1920 ; Martin, 10 juillet 1920, p. 25-27. 
transport était délicate car il fallait éviter toute rupture de charge pour ne pas briser le superphosphate. La solution résida dans l'emploi d'un monorail à traction mixte qui avait été testé dans l'usine de Sète appartenant à la CBPC. L'exemplaire nantais était toutefois doté d'une capacité de transport horaire presque deux fois supérieure - 120-130 tonnes contre 70-80 tonnes - sur un circuit total de 800 mètres $^{38}$.

Le transport automatisé pénétra même à l'intérieur des bâtiments de stockage. Si son usage était connu depuis la fin du XIX ${ }^{e}$ siècle, il était jusqu'alors employé dans les entrepôts sans étage. L'incapacité à faire évoluer un pont roulant dans un édifice à étages multiples fut levée à la fin du conflit dans un entrepôt de Brooklyn, près du port de New York. En 1918, l'intensification de la contribution logistique américaine à l'effort de guerre de ses alliés nécessitait d'abattre le mur de la manutention. Le transport des produits fut entièrement automatisé dans le plus grand des deux bâtiments qui composaient cet entrepôt : long de 300 mètres et large de 93 mètres, il se développait sur une hauteur de dix étages. Les déplacements horizontaux étaient assurés par des chariots porteurs, groupés en rames que remorquaient des tracteurs électriques à accumulateurs. Les déplacements verticaux étaient exécutés soit par monte-charges, soit par ponts roulants. L'auteur de l'article, Victor Sabouret, le meilleur connaisseur français des systèmes de transbordement dans les ports américains, insistait sur l'innovation que représentait l'introduction des tracteurs à accumulateurs dans l'exploitation des grands entrepôts, car ils apportaient " des possibilités nouvelles qui permett[ai]ent de relier convenablement au quai d'accostage des magasins éloignés de 300 à 400 mètres $^{39}$ ». En conclusion, l'ingénieur imaginait d'ailleurs que le site du futur port de Caronte serait tout à fait propice pour accueillir un magasin à étages multiples de type Brooklyn ${ }^{40}$.

38. Silvy-Leligois, 1926, p. 13-14.

39. Sabouret, 1930 , p. 501.

40. La loi du 24 octobre 1919 avait constitué l'acte de lancement officiel des travaux d'aménagement des rives de Port-de-Bouc et de l'étang de Caronte qui étaient les premières annexes du port de Marseille. 


\section{Aspirateurs et pipelines}

Au cours de l'entre-deux-guerres, les ports ont également généralisé l'emploi des pipelines et des aspirateurs. D’après $M$. Croguennec, seul le déchargement du pétrole pouvait être considéré comme satisfaisant avantguerre car c'était " le seul trafic qui avait totalement intégré la notion de fluidité " : c'était le grand avantage du vrac liquide que de pouvoir être transvasé par un système de canalisations depuis les citernes des navires vers les réservoirs à terre ${ }^{41}$. Toutefois, certains ports étaient déjà également équipés en aspirateurs à sucre et céréales. Au cours de la guerre, les aspirateurs pneumatiques se déployèrent dans les ports en raison du rendement supérieur qu'ils offraient par rapport aux systèmes à noria : la partie mobile, réduite à un tuyau flexible, était facile à agencer et le pelletage de la cargaison dans la cale se trouvait réduit au minimum. En 1917, une société de Manchester dota le port de Dunkerque de quatre aspirateurs à grains pour accélérer le ravitaillement de l'armée anglaise ${ }^{42}$. Après le conflit, la chambre de commerce les racheta à l'État dans l'intention d'en faire les premiers éléments d'un équipement intégré de manutention, de stockage et de réexpédition des céréales ${ }^{43}$.

Les appareils pneumatiques se sont vite avérés être un mode de transbordement très efficace pour les céréales, mais aussi pour les oléagineux, le café, le riz ou encore le maïs. À Rouen, des pompes montées sur des pontons flottants permettaient le transfert rapide des céréales en vrac. Une fois chargé, le chaland était immédiatement intégré au train de péniche partant vers Paris. La chambre de commerce équipa la darse sud du bassin des prairies Saint-Gervais d'un appareil pneumatique équipé de deux manches offrant un débit horaire de 120 tonnes. Le grain était automatiquement ensaché et les sacs dirigés vers les wagons stationnant le long de l'élévateur. En plus de la vitesse d'exécution, les aspirateurs offraient l'avantage d'aérer, de dépoussiérer et de débarrasser les grains de leur humidité par leur simple passage dans les tubes, en ne subissant aucune altération ${ }^{44}$.

\footnotetext{
41. Croguennec, 1999.

42. Même si l'on attribue l'invention de l'élévateur pneumatique au colonel du Génie Charles Renard, qui le présenta lors de l'Exposition Universelle de 1878, les Anglais monopolisaient dès avant 1914 la détention des brevets sur ces appareils. Voir Dantin, 1916, p. 17-21.

43. Caufourier, 1925, p. 422-423.

44. Croguennec, 1999.
} 
La même logique prévalut à Bordeaux où, dans l'immédiat après-guerre, une société privée, l'Union commerciale de Bordeaux-Bassens, créa en aval des appontements construits par les Américains trois postes d'accostage dont l'un était dédié au transbordement des céréales. Là aussi, le grain était aspiré par deux appareils pneumatiques mobiles sur rails, qui pouvaient décharger chacun 150 tonnes à l'heure. Les denrées déchargées étaient ensuite placées sur deux transporteurs à courroie surélevés. Les pompes aspirantes qui produisaient le vide dans les canalisations étaient installées dans un bâtiment adjacent aux silos. L'aspiration se produisait à travers des conduites principales de 60 centimètres de diamètre, disposées le long des transporteurs et réunies à d'autres conduites, disposées de part et d'autre de la courroie transporteuse qui longeait l'appontement. Sur ces dernières conduites, des raccords disposés en vingt points différents permettaient de mettre les appareils de déchargement en communication avec la canalisation d'aspiration, par l'intermédiaire de tuyaux souples ${ }^{45}$. Dans l'entredeux-guerres, comme l'a écrit fort justement R. Borruey, la manutention des céréales avait atteint un degré de fluidité proche des dispositifs conçus pour la réception des matières liquides telles que les hydrocarbures.

Une autre marchandise liquide connut une semblable évolution. L'apparition du transport du vin en vrac sur des cargos spécialisés, les pinardiers, entraîna là aussi une adaptation des outils de transbordement $^{46}$. Elle ne fut pas d'ailleurs sans provoquer de fortes résistances chez les dockers. Jusqu'alors, les futailles étaient déchargées au moyen de grues standards dotées de crochets. Comme pour toutes les marchandises conditionnées, l'opération était longue et mobilisait de grandes surfaces de stockage, à l'air libre, sur les terre-pleins. L'apparition des pinardiers entraîna le transfert du vin par pompes, depuis les citernes du navire jusqu'à celles d'un chaland citerne ou bien de réservoirs à terre. Le port de Rouen, qui était l'une des grandes places importatrices de vins d'Afrique du Nord, commença à expérimenter la méthode pour les vins tunisiens au milieu des années 1930. La Société Franco-Algérienne avait construit des entrepôts en arrière des bassins Saint-Gervais d'une contenance avoisinant les 20000 hl. Toutefois, l'usage des pompes ne se généralisa qu'au lendemain

45. "L'installation pour le déchargement... », 1921, p. 530-531.

46. Les pinardiers furent une particularité de la flotte marchande française. Leur essor date de la deuxième moitié des années 1930. Voir Lenhof, 2005, p. 490-491. 
de la Deuxième Guerre mondiale, au moment de l'âge d'or des pinardiers, dans les années 1950 et $1960^{47}$.

\section{Les entrepôts spécialisés}

En même temps que les innovations dans l'outillage de transbordement, de nouveaux types de bâtiments de stockage apparurent à la fin du XIX ${ }^{e}$ siècle. Un nouveau système technique se mit alors en place pour répondre aux nouvelles injonctions de l'économie des transports maritimes. Les docks-entrepôts qui constituaient la matrice du modèle des " ports à l'anglaise " étaient déclassés au profit d'un autre modèle en phase avec le mode de production tayloro-fordien ${ }^{48}$. Les cargos, les engins de transbordement et les entrepôts connurent une dynamique de spécialisation concomitante. Les bâtiments furent de plus en plus conçus comme des unités dédiées à un fret spécifique, opérant son magasinage et / ou son traitement.

Ainsi, les nouvelles techniques de déchargement des céréales ont donné naissance aux silos modernes. Ils ont été, par exemple, le premier système complet d'outillage spécialisé dont s'est doté le port de Marseille. Le stockage du grain posait des problèmes d'humidité et de surchauffe. Dans les magasins traditionnels, les blés étaient donc étalés sur des planchers et régulièrement pelletés pour les aérer. L'autre solution résidait dans l'ensachage du grain, ce qui évitait le pelletage et améliorait les conditions de conservation, mais elle était plus onéreuse pour le consignataire. L'ensilage moderne reposait sur de nouveaux principes. Les grains étaient tout d'abord déversés par gravité depuis le sommet du bâtiment, après avoir été drainés par des transporteurs à courroie, des élévateurs à godets ou des aspirateurs depuis les navires. Il y avait une contrainte, en revanche. Les murs devaient associer des qualités de parfaite étanchéité, d'épaisseur et de robustesse, tant pour résister aux poussées internes des cuves remplies que pour assurer leur isolation thermique. Le béton armé apporta la solution. La Compagnie des Docks construisit son premier silo au milieu des années 1920. Elle fut imitée par la Société Générale de Transbordements Maritimes (SGTM) qui mit le sien en service en 1928. Situé en arrière du bassin de la Pinède, le silo de la Madrague, d'une contenance de 30000 tonnes, était d'une capacité d'un tiers supérieure au premier. Pour dépasser

47. Croguennec, 1999.

48. Marnot, 2020, p. 201-204. 
sa rivale, la Compagnie des Docks fit édifier un deuxième petit silo de 13000 tonnes. Cette « bataille des silos » entre les deux firmes avait pour enjeu la captation du trafic céréalier ${ }^{49}$.

Cette dernière était d'autant plus cruciale qu'il fallait rentabiliser ces équipements modernes. Ainsi, le silo de la SGTM était un bâtiment d'une centaine de mètres de longueur sur plus de 20 mètres de large. Le sommet de la tour des élévateurs s'élevait à près de 60 mètres au-dessus du niveau de la mer. À l'intérieur, les cellules à grains avaient des contenances respectives de 100, 250 et 500 tonnes. Un plancher permettait l'ensachage des céréales qui pouvaient être chargées directement sur camions ou sur wagons, le silo étant relié par embranchement particulier à la gare d'Arenc. Du côté quai, le grain était déchargé au moyen de deux aspirateurs pneumatiques assurant chacun un débit horaire de 30 tonnes. Côté terre-plein, la livraison sur camions était exécutée au moyen de dalles glissières fixes, capables de charger en cinq minutes un camion de 5 tonnes. Pour la mise en wagons, les sacs ou le grain en vrac tombaient sur des transporteurs situés sous le plancher d'ensachage qui était percé de trappes ${ }^{50}$.

Les entrepôts frigorifiques constituaient l'autre édifice symbolique de l'architecture portuaire de l'entre-deux-guerres. Ils étaient destinés au stockage des denrées périssables. Inventé au début des années 1870, le navire frigorifique fut d'abord utilisé sur les routes Amérique du Sud-Europe, d'un côté, et Océanie-Europe, de l'autre, afin de fournir les consommateurs britanniques en viandes d'Argentine, d'Australie et de Nouvelle-Zélande. Cela explique que la Grande-Bretagne, la première, mit en service la plus importante flotte de cargos frigorifiques et que ses villes portuaires disposèrent d'une capacité de stockage de denrées froides sans équivalents. Au moment du conflit, Londres, par exemple, disposait de 33 entrepôts frigorifiques et Liverpool de 18. Avec une capacité de stockage six fois inférieure, la France était loin derrière ${ }^{51}$. Mais la guerre donna un élan à l'importation de viandes frigorifiées, parce que l'agriculture française n'avait tout simplement plus la capacité de fournir le pays en viande fraîche. Aussi le Conseil supérieur des Travaux Publics préconisa-t-il la création de vastes établissements à Marseille, Le Havre et Bordeaux. L'entrepôt de La Pallice-La Rochelle,

49. En 1934, elles finirent par conclure un accord amiable sur les tarifs et de partage des arrivages, afin de maintenir la rentabilité de leurs installations qui avaient coûté très cher. Voir Borruey, 1994. 50. Fèvre, 1933.

51. Gouault, 1919. 
entièrement construit en ciment armé, fut mis en service en 1918. Il servit au stockage des viandes importées d'Argentine pour satisfaire les besoins en protéines des soldats et secondairement ceux de la population civile. Les viandes étaient réceptionnées au rez-de-chaussée puis emmenées sur des chariots jusqu'aux monte-charges. La rapidité du transfert était une condition sine qua non pour éviter la rupture de la chaîne du froid. Aussi l'acheminement des marchandises dans les chambres situées dans les étages supérieurs était-il assuré par deux ascenseurs de 1,4 tonne de charge utile par appareil et par un transporteur Burton. Les ascenseurs étaient placés au centre des chambres, qui rayonnaient autour d'eux. Les viandes débarquées étaient déposées dans les chambres moins de dix minutes après leur sortie des cales. À l'intérieur, les manutentions humaines étaient évitées autant que possible. Par exemple, la circulation des denrées au sein de l'entrepôt du Havre était assurée par un monorail aérien, mû bien sûr à l'électricité. Son réseau couvrait deux kilomètres le long desquels étaient répartis plus de 200 aiguillages et plaques d'évolution ${ }^{52}$.

Chacun des cinq étages de l'entrepôt rochelais était équipé d'une chambre froide dont la température oscillait entre $-8^{\circ}$ et $-11^{\circ} \mathrm{C}$. Le froid était obtenu par l'emploi de l'anhydride carbonique. Par rapport aux méthodes utilisant l'ammoniaque ou l'anhydride sulfureux, ce procédé présentait l'avantage de ne pas dégager d'odeur. En outre, intervenant dans la végétation des plantes, son usage était particulièrement recommandé pour l'entreposage des fruits et des légumes. Une fuite de gaz dans la chambre n'aurait ainsi eu aucun effet sur les fruits entreposés. Toutefois, la conservation des œufs, des fruits et des légumes était minoritaire par rapport à ce qui demeurait la vocation essentielle de l'entrepôt frigorifique de La Pallice, à savoir le stockage des bœufs et moutons argentins. Seule une partie des chambres froides servit à l'entreposage des beurres fabriqués dans les coopératives du Poitou et de Charente. Il fut constaté que même après une année de conservation la qualité du beurre n'était pas altérée ${ }^{53}$. Ses capacités de conservation de longue durée présentaient in fine des vertus économiques : le frigorifique apparut aussi comme un organe de régulation pour

52. Pawlowski, 9 juin 1928, p. 557.

53. On alla le plus loin possible dans l'automatisation des opérations puisqu'un appareil enregistrait l'intégralité des colis pénétrant dans les docks, tandis que d'autres, placés sur les monorails, immatriculaient le poids des arrivages. Pawlowski, 30 juin 1928, p. 638-639. 
empêcher l'avilissement des prix et l'encombrement des marchés, lors des périodes de surabondance.

L'exemple rochelais rappelle que les entrepôts frigorifiques sont spécialisés dans une forme de conservation, mais qu'ils sont polyvalents quant au stockage des produits. Ainsi, à Bordeaux, le frigorifique installé sur le site de Bassens au lendemain de la Première Guerre mondiale servit initialement à l'entreposage de la viande importée d'Argentine. Cependant, l'augmentation des droits de douanes sur la viande congelée en 1928 entraîna la disparition de ce courant de trafic. La Société des Entrepôts Frigorifiques et Docks de la Gironde (SEFDG) compensa en partie cette perte par la conservation des morues, dont la pêche et le séchage constituaient une très vieille activité du port et plus exactement de la commune de Bègles, située en amont. La SEFDG loua une des quatre chambres froides de son entrepôt, ce qui permettait de conserver la morue avant son traitement dans l'usine de préparation créée à proximité. Les installations des Pêcheries et Sécheries de Bordeaux comprenaient un hall de dessalage et de lavage mécanique, une sécherie à l'air chaud, une sécherie d'été au soleil et un atelier de préparation du poisson $\sec ^{54}$.

L'entrepôt frigorifique de Bordeaux fut aussi de plus en plus alimenté par le trafic de la banane, qui prit un essor remarquable au cours de l'entre-deuxguerres. Le cargo bananier devint d'ailleurs le navire fruitier emblématique des années 1930. Le port de Rouen inaugura ce nouveau type de trafic avec les Antilles françaises dès le lendemain de la guerre. Or le traitement de ce fruit fragile était délicat et nécessitait, par conséquent, la mise en place d'appareils de manutention et de hangars spéciaux. Quatre portiques portant une chaîne continue à poches assuraient le transfert des régimes de bananes depuis la cale vers le hangar. Les cargaisons y pénétraient par l'intermédiaire de panneaux aménagés dans le toit. Les appareils étaient entièrement clos pour protéger les fruits contre la chaleur et les intempéries. L'étanchéité générale de la chaîne de transbordement, à la sortie des cales comme pendant la traversée des toitures, avait pour but d'éviter les écarts de températures. D'un débit de 1500 à 1600 régimes à l'heure, les installations rouennaises pouvaient décharger en deux jours les plus 
grands navires bananiers pouvant remonter jusqu'au port et contenant en moyenne 80000 régimes $^{55}$.

\section{Conclusion}

La nouvelle poussée de mécanisation des opérations de manutention dans les ports de commerce au cours de l'entre-deux-guerres invite à formuler quatre types de réflexions. La première consiste à souligner la très nette propension, dans la manipulation des vracs, à l'automatisation des tâches entre le navire, l'entrepôt, les différents ateliers et les espaces de pré et post-acheminement terrestres. S'affirma alors une convergence entre spécialisation du navire, celle des engins de transbordement et celle des bâtiments de stockage. C'est in fine la spécialisation de l'espace de travail portuaire qui se trouvait au bout de cette logique et donc celle du terminal. S'en dégageait le principe de poste de travail qui était bien la marque du modèle du port tayloro-fordien, coextensif à la deuxième révolution industrielle $^{56}$. Sur le plan énergétique, l'électricité triompha d'ailleurs comme source de force motrice.

La dynamique de mécanisation intégrale du transbordement des vracs ne fut pas, au demeurant, l'apanage des grands ports de commerce. À Lorient, par exemple, le nouveau port de commerce de Kergroise était principalement constitué d'un terminal charbonnier au dispositif de déchargement automatisé. On constate une semblable dynamique de continuum technique dans les ports de pêche industrielle. Dans le bassin des Chalutiers de La Rochelle s'est mis en place, au cours de l'entre-deux-guerres, une chaîne de déchargement du poisson, de son traitement et de son expédition par wagons isothermes ${ }^{57}$.

Le troisième point saillant dépasse le seul cadre de la manipulation des pondéreux. De manière plus générale, la marche à l'automatisation du transfert des marchandises fut une tendance lourde de l'entre-deux-guerres. Elle fut appliquée aussi, on l'a vu, à la manutention de certaines matières premières conditionnées comme les viandes. Les marchandises diverses représentaient encore un verrou technique, mais les premières expériences

55. Croguennec, 1999.

56. Marnot, 2020.

57. Le Bouëdec, Cérino, 2015 ; Moulinier, chapitre III. 
de mise en boîte dans ce qu'on appelait alors des " cadres de classement ", furent menées par les compagnies ferroviaires ${ }^{58}$. Tout comme le portique, le principe du conteneur existait bien avant 1940. Lassociation des deux serait réalisée seulement quelques décennies plus tard. Par ailleurs, des innovations furent introduites pour réduire, voire annihiler, la séquence de rupture de charge dans le transport des voyageurs, à l'image du train-ferry mis en circulation sur la ligne Dunkerque-Douvres à partir de $1936^{59}$.

La dernière réflexion se situe à l'intersection de l'histoire technique et de l'histoire sociale. Elle concerne bien évidemment le jeu complexe des relations entre automatisation du transbordement et travail des dockers. Elle renvoie à la question de la réception des nouveaux engins de transbordement sur les quais par le personnel de manutention, à l'évolution de l'effectif là où la mécanisation devient envahissante, ainsi qu'à la redéfinition du contenu du travail même des dockers face au changement technique.

\section{Sources}

Caufourier P., «Le silo à grain du port de Dunkerque ", Le Génie civil, n 18 , 2 mai 1925.

Caufourier P., "Les ferry-boats de la ligne de Dunkerque à Douvres", Le Génie civil, n² 21, 21 novembre 1936.

Claveille Albert, «Rapport au Président de la République relatif aux mesures prises en 1918 dans les ports maritimes en vue de faire face aux importations ", 21 janvier 1919, Annales des Ponts et Chaussées, 1919.

Dantin C., "Le transport pneumatique des matières en grains et principalement des céréales ", Le Génie civil, n 2, 8 janvier 1916.

FÈvre L., "Le silo à céréales de la Société Générale de Transbordements Maritimes, à Marseille ", Le Génie civil, n 1, 1 1er juillet 1933.

Gouault E., "L'outillage frigorifique et le commerce des viandes frigorifiées ", Le Génie civil, nº 18, 3 mai 1919.

"Grues à volée variable à bord des navires ", Le Génie civil, 30 juillet 1938.

"Grues sur portique pour le chargement des minerais au port de Nantes, Le Génie civil, 5 novembre 1932.

HeRsent Georges, Les grands ports français, leur transformation et leur autonomie, Imprimerie Chaix, Paris, 1908.

58. Borde, 2015.

59. Caufourier, 1936, p. 456-458 ; Starostina, 2010. 
Hersent Georges, "Notre outillage maritime ", dans L'outillage économique de la France, Librairie Félix Alcan, Paris, 1921.

Kauffman A., "Les installations nouvelles exécutées pendant la guerre dans le port de Nantes et la Loire maritime ", Le génie civil, n 4, 24 janvier 1920.

Laroche Charles et Pobeguin M.-E., "Étude comparée de l'outillage des ports français et étrangers", dans Actes du quatrième Congrès national des travaux publics, Association française pour le développement des travaux publics, Paris, 1912, p. 1-11.

LARoche Charles, "L'évolution des travaux maritimes de 1830 à 1930 ", Annales des Ponts et Chaussées, "Mémoires et Documents ", nº 9, t. 1, 1931.

"L'installation pour le déchargement et la manutention des grains à Bordeaux-Bassens ", Le Génie civil, n² 25, 17 décembre 1921.

"L'outillage mécanique des ports. Les appareils de manutention des minerais et des charbons aux États-Unis ", Le Génie civil, n 7, 14 février 1920.

Martin Henry, "Les nouvelles installations du port de Bordeaux ", Le Génie civil, $\mathrm{n}^{\circ} 1,3$ juillet 1920 .

Martin Henry, "Les nouvelles installations de déchargement du port de Bordeaux ", Le Génie civil, n² 2, 10 juillet 1920.

PaWLOwski Auguste, "Les ports français et la guerre ", Le Génie civil, n² 21, 24 novembre 1917.

Pawlowski Auguste, "Les ports français et la guerre-Dunkerque ", Le Génie civil, $\mathrm{n}^{\circ} 22,31$ mai 1919.

Pawlowski Auguste, "L'entrepôt de la Société des Docks Frigorifiques du Havre ", Le Génie civil, n 23, 9 juin 1928.

Pawlowski Auguste, "L'entrepôt frigorifique du port de La Pallice, près de La Rochelle », Le Génie civil, n² 26, 30 juin 1928.

Pawlowski Auguste, "L'entrepôt frigorifique de Bordeaux-Bassens », Le Génie civil, nº 8,23 février 1929.

Sabouret Victor, "Aménagement de l'outillage et des voies à l'arrière de quelques postes maritimes intensifs ", Annales des Ponts et Chaussées n 5, 1919.

SABouret Victor, "L'emploi du pont roulant dans les grands entrepôts maritimes ", Le Génie civil, n 21, 24 mai 1930.

Silvy-Leligois J., "La manutention mécanique des superphosphates à l'usine de Nantes de la Compagnie bordelaise des produits chimiques ", Le Génie civil, $\mathrm{n}^{\circ} 14,2$ octobre 1926. 
Viguerie Charles, « Note sur l'outillage des ports ", Actes du quatrième Congrès national des travaux publics français, Association française pour le développement des travaux publics, Paris, 1912.

\section{Bibliographie}

Barbance Marthe, Saint-Nazaire. Le port, la ville, le travail, Marseille, 1949 (Laffitte Reprints, 1979, réédition).

Borde Christian, "La genèse du système des containers, entre route, rail et navigation maritime (1896-1956) ", dans EcK Jean-François, Tilly François, Touchelay Béatrice (dir.), Espaces portuaires. L'Europe du Nord à l'interface de économies et des cultures, $19^{\circ}$ et $20^{\circ}$ siècle, Presses du Septentrion, Villeneuve d'Ascq, 2015, p. 147-158.

Borruey René, Le Port moderne de Marseille, du dock au conteneur, 1844-1974, t. IX, Histoire du commerce et de l'industrie de Marseille, XIX'-XX' siècles, CCIM, Marseille, 1994.

Cassagne Bernard, Naissance et développement d'une industrie originale, les sécheries de morue de l'agglomération bordelaise (1825-1990), thèse de doctorat, Université Bordeaux 3, dir. A. Fernandez, 3 vol., 2015

Croguennec Michel, L'aménagement du port de Rouen de 1800 à 1940 : contraintes, techniques et stratégies, thèse de doctorat, Université de Rouen, dir. Michel Pigenet, 1999.

Landes David, L'Europe technicienne ou le Prométhée libéré, Gallimard, Paris, 1975.

LE BOUËDEC Gérard, CÉRINo Christophe, Lorient, ville portuaire. Une nouvelle histoire des origines à nos jours, PUR, Rennes, 2017.

Lenhof Jean-Louis, Les hommes en mer. De Trafalgar au Vendée Globe, Armand Colin, Paris, 2005

MaRnot Bruno, Les grands ports de commerce français et la mondialisation au XIX siècle, PUPS, Paris, 2011.

Marnot Bruno, Le refus du déclin. Le port de Bordeaux au XIXe siècle, PUB, Pessac, 2012

Marnot Bruno, Les villes portuaires maritimes en France, XIX'-XXe siècle, Armand Colin, Paris, 2015

Marnot Bruno, "Pour une histoire des modèles techniques portuaires ( $\mathrm{XIX}^{\mathrm{e}}$ $\mathrm{XxI}^{\mathrm{e}}$ siècles)", Revue d'Histoire Maritime, $\mathrm{n}^{\circ}$ 28, 2020, p. 193-205. 
Moulinier Henri, La pêche industrielle de La Rochelle. Les grands chalutiers (1871-1914), PUR, Rennes, 2015.

Musso Alain, La religion industrielle, monastère, manufacture, usine. Une généalogie de l'entreprise, Fayard, Paris, 2017.

Mumford Lewis, Technique et civilisation, La Découverte, Paris, 2016 (édition originale 1934).

Starostina Natalia, "Le Train-Ferry Douvres-Dunkerque, le Train Bleu et autres initiatives intermodales dans la France de l'entre-deux-guerres ", Revue d'Histoire des Chemins de fer, ${ }^{\circ} 42-43,2010$, p. 49-65.

\section{L'auteur}

Bruno Marnot est professeur d'histoire contemporaine à l'Université de La Rochelle, membre de l'UMR LIENSs et directeur-adjoint du GIS Histoire et Sciences de la Mer. Il travaille sur l'histoire technique et économique des ports de commerce aux $\mathrm{xIX}^{\mathrm{e}}$ et $\mathrm{xx}$ siècles. Il a récemment publié " Pour une histoire des modèles techniques portuaires (xIx ${ }^{\mathrm{e}}$-xxle siècles) ", Revue d'Histoire Maritime, $n^{\circ} 28,2020$, p.193-205. Contact : bruno.marnot@univ-lr.fr ; www.histoiresciences-mer.org. 